\title{
TREATMENT OF LEUKEMIA AND RELATED DISORDERS WITH 6-MERCAPTOPURINE
}

\author{
By F. H. Bethell and D. S. Thompson \\ University of Michigan, Ann Arbor, Mich.
}

Since February 1953, 48 patients afflicted with leukemia and allied disorders have received 6-mercaptopurine at the University of Michigan Hospital and at the Simpson Memorial Institute. Eight of the patients died within three weeks of the time of institution of therapy, most of them receiving the drug for less than one week. The remaining 40 serve as the clinical material for the analysis of our therapeutic results. It is recognized that for comparisons of antileukemia agents from the standpoint of survival, all cases should be included. However, such a comparison is not possible for our series because of previous or subsequent use of agents other than 6-mercaptopurine in many instances. It is our purpose to evalute the nature of the response obtained with 6-mercaptopurine in those patients who received the drug alone for a sufficient time to permit observation of its effect.

Our practice is largely confined to adults, and in the series of 40 cases only five patients were under 15 years of age. It soon became evident that our better therapeutic responses were being obtained in younger adults, and that the most satisfactory dividing line appeared to be at 35 . This is an arbitrary separation, of course, but one cannot help being impressed with the inability of the middle-aged and elderly patient having acute leukemia to restore any degree of normal hemopoiesis, even though leukemic cell proliferation is suppressed.

Factors which influence the responsiveness of a case of leukemia to specific medication, include, in addition to the age of the patient, the cell type, the proliferative activity, and the degree of dedifferentiation. Duration of the disease before institution of treatment bears a relation to the therapeutic response, but this variable cannot be controlled or readily defined, and it may be expected to affect more or less equally the several categories of acute leukemia.

The rapidly proliferating leukemias are classed as acute. The most undifferentiated type is the stem cell or hemocy toblastic. Those showing some cellular differentiation are the lymphocytic, granulocytic, and monocytic. Less rapidly proliferating forms of leukemia are classed as subacute. Most subacute leukemias are granulocytic, and this type only is represented in the present series. Chronic leukemias show variable rates of cell proliferation and, until they become advanced, exhibit a relatively high degree of cell differentiation. In addition to these forms there are the disseminated lymphocytic sarcoma and reticulum cell sarcoma, both of which may be leukemic. Employing the criteria for good and partial remissions suggested for the conference on 6-mercaptopurine on which this monograph is based, our results may be summarized as follows:

There were three cases of acute hemocytoblastic leukemia, all under 35 years of age. Two of these patients had good remissions, and one a partial remission. 
There were four cases of acute lymphocytic leukemia. Of three patients under 35, two had good remissions and one a partial remission. One patient over 35 had a partial remission.

The series includes 10 cases of acute granulocytic leukemia. Of the seven patients under 35, two had good remissions, four had partial remissions, and in one there was no favorable response. Of the three patients 35 years of age or over, one had a partial remission and two had no responses.

A total of four cases of acute monocytic leukemia were treated. Two of these patients were under 35; the one had a partial remission and the other no remission. The two patients who were over 35 failed to respond to 6-mercaptopurine.

There were six cases of subacute granulocytic leukemia. Of the two patients under 35, the one had a partial remission and the other no remission. Four patients 35 or over included three who had partial remissions and one who had no remission.

Six patients having chronic granulocytic leukemia, all in the terminal acute stage of the disease, were treated with 6-mercaptopurine. Two were under 35, and four were 35 or older. All six patients had remissions which, from a hematologic standpoint, must be classed as partial, but clinically these remissions were good to excellent, and they lasted from a few weeks to several months.

The series includes six cases of disseminated lymphocytic sarcoma, three under and three over 35 years. Two of the younger group had good remissions and one a partial remission. Of those over 35 , two had partial remissions and one no remission.

One patient over 35 years of age afflicted with wide-spread reticulum cell sarcoma obtained a partial remission while receiving 6-mercaptopurine.

Discussion. The number of patients in each category is small, so that interpretation of the data must be regarded as tentative. The results so far obtained with 6-mercaptopurine indicate that favorable responses may be obtained in a high percentage of children and of young adults having acute leukemia. The most favorable effects, in our experience, have been observed in patients suffering from acute hemocytoblastic and acute lymphocytic leukemia. Least beneficial results were obtained in monocytic leukemia. In all of our cases of acute leukemia under the age of 35 , the percentage incidence of good remissions was 28.6, whereas the incidence of partial remissions was 33.3 per cent. Over the age of 35 there were no good remissions, and only 2 partial remissions occurred among the six patients treated.

In the less actively proliferative subacute leukemia of granulocytic type, no good remissions were obtained. Although patients having this form of the disease may be partially controlled and may have protracted illnesses, substantial hematologic and clinical improvement has not been observed in any of our cases.

6-Mercaptopurine is an exceedingly useful drug, in our experience, in the management of the late stage of chronic granulocytic leukemia when other forms of therapy are no longer effective. A sense of well-being is usually temporarily restored to such patients, and the extreme hypermetabolism and apprehensiveness of the terminal phase of this disease is largely averted. 
The compound may be of considerable value in the treatment of disseminated lymphosarcoma and reticulum cell sarcoma.

No specific toxic effects, other than those on bone marrow, have been noted in association with 6-mercaptopurine therapy. Megaloblastoid transformation of erythropoiesis, such as may occur as a result of administration of folic acid antagonists, has rarely been observed after 6-mercaptopurine therapy. However, prolonged administration, for at least four or five weeks, is accompanied by a peculiar change in the morphology and staining reaction of the nucleated erythroid elements in the marrow. The early normoblasts show a loss of normal nuclear chromatin architecture which can best be described as karyolysis. The chromatin strands swell, lose their normal distinct ropelike pattern and become hemogeneous opaque masses. Simultaneously there may be a great increase in the total number of nucleated erythrocytes.

Conclusions. Forty-eight patients afflicted with leukemia were treated with 6-mercaptopurine. The series included acute, subacute, and advanced chronic leukemia and disseminated malignant lymphoma. Evaluation of therapy is based on data obtained on 40 patients who were observed for at least three weeks.

Dosage of 6-mercaptopurine was within the range of 2.0 to $3.0 \mathrm{mgm}$. per kilo of body weight daily for initial therapy, and a total of 25 to $50 \mathrm{mgm}$. daily for maintenance in adult cases.

The results indicate that 6-mercaptopurine has a limited value in the treatment of acute leukemia, other than monocytic, in children and young adults. It is useful as palliation in the terminal stage of chronic granulocytic leukemia, and its administration may be temporarily beneficial in disseminated malignant lymphoma.

The combined or sequential use of 6-mercaptopurine with other antimetabolites and with cortisone and ACTH has, in general, improved the outlook of patients having acute leukemia. 